\title{
Bolt Detection Signal Analysis Method Based on ICEEMD
}

\author{
Chunhui Guo, ${ }^{1,2}$ Zhan Zhang $\mathbb{D}^{1},{ }^{1}$ Xin Xie, ${ }^{3}$ and Zhengyu Yang ${ }^{3}$ \\ ${ }^{1}$ College of Water Conservancy and Hydropower Engineering, Hohai University, Nanjing, China \\ ${ }^{2}$ Key Laboratory of Hydraulic and Waterway Engineering of the Ministry of Education, Chongqing Jiaotong University, \\ Chongqing, China \\ ${ }^{3}$ Department of Electrical and Computer Engineering, Northeastern University, Boston, MA, USA
}

Correspondence should be addressed to Zhan Zhang; zhanzhang_hhu@qq.com

Received 23 September 2017; Revised 9 January 2018; Accepted 28 February 2018; Published 18 April 2018

Academic Editor: Carlo Trigona

Copyright (C) 2018 Chunhui Guo et al. This is an open access article distributed under the Creative Commons Attribution License, which permits unrestricted use, distribution, and reproduction in any medium, provided the original work is properly cited.

The construction quality of the bolt is directly related to the safety of the project, and, as such, it must be tested. In this paper, the improved complete ensemble empirical mode decomposition (ICEEMD) method is introduced to the bolt detection signal analysis. The ICEEMD is used in order to decompose the anchor detection signal according to the approximate entropy of each intrinsic mode function (IMF). The noise of the IMFs is eliminated by the wavelet soft threshold denoising technique. Based on the approximate entropy and the wavelet denoising principle, the ICEEMD-De anchor signal analysis method is proposed. From the analysis of the vibration analog signal, as well as the bolt detection signal, the result shows that the ICEEMD-De method is capable of correctly separating the different IMFs under noisy conditions and also that the IMF can effectively identify the reflection signal of the end of the bolt.

\section{Introduction}

The bolt anchoring system is subject to the geological conditions and the construction technology effect. If there are any hidden dangers that have not been detected, then it will cause engineering accidents and serious economic losses. Therefore, the construction quality of the bolt anchorage must be checked, so as to ensure the safety of the project. During the early stage, the detection of the anchor's anchoring quality is mainly based on the drawing test [1-3]. However, this method will cause damage to the anchoring system. The method is also not suitable for large-scale detection and cannot be fully reflected [4-6].

The detection method for the quality of the anchor will be gradually replaced by the use of nondestructive testing methods, such as the acoustic wave method [7-10]. This method is established based on the mathematical model of the one-dimensional elastic rod $[8,11,12]$. The assumption is that the longitudinal wave wavelength that is generated by the exciting force is much larger than that of the bolt radius, so the transverse displacement of the system can be neglected. By solving the longitudinal one-dimensional wave equation, the dynamic response of the bolt system is obtained.
The low-end reflection signal of the bolt can be easily disturbed during the process of bolt detection; it is difficult to directly obtain the reflected wave arrival time. In order to obtain the effective signal, many data processing methods, such as the short-time Fourier transform, the Gabor transform, the Wigner-Ville transform, and the wavelet transform, are proposed. Wavelet transform is the most used signal analysis method among them [13-17]. However, the effect of the wavelet transform is often limited by the wavelet base, as well as the number of decomposed layers.

The empirical mode decomposition (EMD) can adaptively select the substrate according to the characteristics of the signal for the multiresolution analysis of the signal, which will overcome the wavelet base selection problem [18-20]. The decomposition is based on the local timescale of the data. There have been many applications about EMD processing detection signal [21-24]. However, the EMD encounters some modal aliasing problems during the processing procedure [25-27]. The ensemble empirical mode decomposition (EEMD) overcomes the modal aliasing problem that is inherent of the EMD, but due to the addition of different white noise, the decomposition may produce a false mode, which can also cause errors. The reconstructed signal still 
includes residual noise, and different realizations of signal and noise may produce different modes [28-30]. Complete EEMD (CEEMD) has been successfully applied to seismic signal analysis. Li et al. used CEEMD to obtain an exact reconstruction of the original signal and a better spectral separation of the modes with synthetic and real seismic data [31]. However, the CEEMD cannot be proven, and the final averaging problem remains unsolved since different noisy copies of the signal can produce a different number of modes [32]. In recent years, the improved complete EEMD (ICEEMD) has been proposed by adding adaptive white noise to the signal and by redefining the calculations of the local mean for each model [29, 32-35]. The result shows that the method is superior to the traditional method. Although EMD is more adaptive and more efficient, the EEMD outperforms EMD in causing less mode mixing [36]. The CEEMD outperforms EMD in causing less mode mixing and EEMD in better reconstruction performance [37]. The ICEEMD, as illustrated in the paper, outperforms CEEMD in being more physical meaningful and less number of components.

Based on the mentioned research, the ICEEMD method is introduced into the bolt detection signal analysis in this paper. However, the actual signal of bolt detection is under noise interference. The processing signal under noise is critical problem with ICEEMD for bolt detection. By combining the approximate entropy and the wavelet denoising principle, the ICEEMD-De was established based on the ICEEMD. Then, the ICEEMD-de was used to process the simulation vibration signal and the actual bolt detection signal.

\section{Theory and Methodology}

Based on the ICEEMD anchor detection signal analysis method, the ICEEMD-De integrates ICEEMD, the approximate entropy, and wavelet denoising. The three methods are introduced in the section.

2.1. ICEEMD Principle. The ICEEMD method is able to effectively prevent the occurrence of false IMF by adding the adaptive white noise to the signal and by redefining the local mean of each modal. Assuming the anchor detection signal $s$, then the decomposition process of the ICEEMD is as follows.

(1) The signal $s$ is added to the M group Gaussian white noise in order to generate a new signal $s^{i} . s^{i}$ can be expressed as

$$
s^{i}=s+\beta_{k} w^{i}
$$

where $w^{i}(i=1,2, \ldots, M)$ is one group of Gaussian white noise, $\beta_{k}=\varepsilon_{0} \operatorname{std}\left(r_{k}\right), r_{k}$ is the $k$ th residue, $\varepsilon_{0}$ takes 0.2 .

(2) The $k$ th mode can be obtained by EMD. We can obtain the mean of the $k$ th mode and have

$$
\left\langle E_{k}\left(s^{i}\right)\right\rangle=s^{i}-\left\langle M\left(s^{i}\right)\right\rangle,
$$

(3) $s^{i}$ is decomposed by using EMD. We obtain 1th residue $r_{1}$ and 1st IMF $d_{1}$. We have

$$
\begin{aligned}
r_{1} & =\left\langle M\left(s^{i}\right)\right\rangle, \\
d_{1} & =s-r_{1} .
\end{aligned}
$$

(4) We take 2 nd residue, $r_{2}$, as the local mean of $r_{1}+$ $\beta_{1} E_{2}\left(w^{i}\right)$. The 2 nd IMF $d_{2}$ is

$$
\begin{aligned}
& r_{2}=\left\langle M\left(r_{1}+\beta_{1} E_{2}\left(w^{i}\right)\right)\right\rangle, \\
& d_{2}=r_{1}-r_{2} .
\end{aligned}
$$

(5) For any $r_{k}$ and $k$ th IMF $d_{k}$, the expression is as follows:

$$
\begin{aligned}
& r_{k}=\left\langle M\left(r_{k-1}+\beta_{k-1} E_{k}\left(w^{i}\right)\right)\right\rangle, \\
& d_{k}=r_{k-1}-r_{k} .
\end{aligned}
$$

Go to step (3); we obtain all of the IMF.

2.2. Approximate Entropy. All of the IMF approximate entropy can be expressed as $\{A\}=\left\{A_{1}, A_{2}, \ldots, A_{k}\right\}$. Then the calculation procedure $A_{k}$ is as follows.

(1) Take $k$ th IMF as the time series of $n$ points and define it as

$$
\{Z\}=\left\{z_{1}, z_{2}, \ldots, z_{n}\right\}
$$

(2) Compute the binary distance matrix $B$ of the time series:

$$
B=\left[\begin{array}{cccc}
b_{11} & b_{12} & \cdots & b_{1 n} \\
b_{21} & b_{22} & \cdots & b_{2 n} \\
\vdots & \vdots & & \vdots \\
b_{n 1} & b_{n 2} & \cdots & b_{n n}
\end{array}\right]
$$

where

$$
b_{i j}= \begin{cases}1, & \left|z_{i}-z_{j}\right|<a \\ 0, & \left|z_{i}-z_{j}\right| \geq a \\ 1, & \end{cases}
$$

$a$ is threshold ( $a=0.1 \sim 0.2)$ [38].

(3) Compute the ratio of $n+m+1$ and $n-m+2$ to number for $B$ matrix element less than $a C_{i}^{2}$ and $C_{i}^{3}$ as follows:

$$
\begin{aligned}
C_{i}^{2} & =\sum b_{i j} \cap b_{(i+1)(j+1)}, \quad j=1,2, \ldots, n-1, \\
C_{i}^{3} & =\sum b_{i j} \cap b_{(i+1)(j+1)} \cap b_{(i+2)(j+2)},
\end{aligned}
$$

where \langle\rangle is the operator of mean.

$$
j=1,2, \ldots, n-2 .
$$




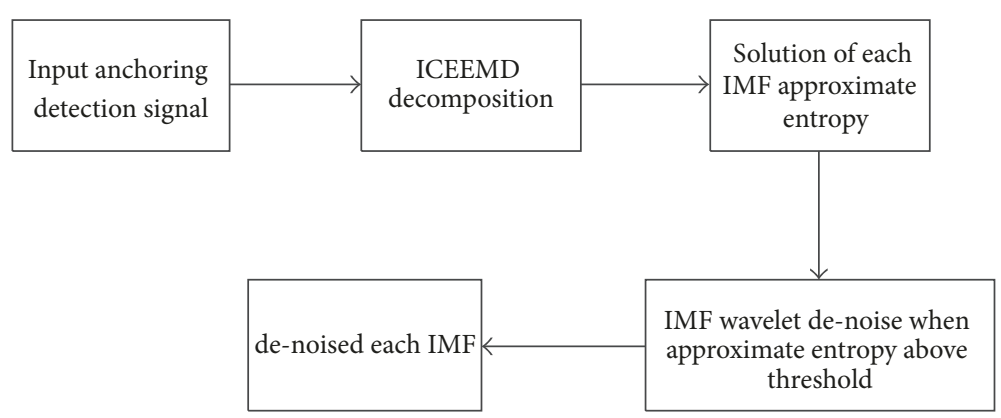

FIGURE 1: Anchoring detection signal analysis flowchart using ICEEMD-De.

(4) Compute $C_{i}$ of the nature logarithm, and get the average of $C_{i}$ of the nature logarithm. Then the approximate entropy of the $k$ th IMF $A_{k}$ is as follows:

$$
\begin{aligned}
& \Phi_{1}=\frac{1}{n-m+1} \sum_{i=1}^{n-m+1} \operatorname{In} C_{i}^{2}, \\
& \Phi_{2}=\frac{1}{n-m+2} \sum_{i=1}^{n-m+1} \operatorname{In} C_{i}^{3}, \\
& A_{k}=\Phi_{1}-\Phi_{2} .
\end{aligned}
$$

(5) The wavelet denoise is performed with the approximate entropy of the IMF that is greater than the threshold.

2.3. Wavelet Denoise. The wavelet denoise is achieved based on a critical threshold. The main steps of its denoise principle are as follows.

(1) Select the appropriate wavelet base and the number of decomposition layers. We take the wavelet transform with the noise signal $s$ and obtain its wavelet coefficients $w^{j}$ :

$$
\begin{aligned}
v^{j-1} & =D_{0} H v^{j}, \\
w^{j-1} & =D_{0} G v^{j},
\end{aligned}
$$

where $H$ is the low-pass filter, $G$ is the high-pass filter, $v$ is for the scale factor, and $w$ is the wavelet coefficient.

(2) Select the appropriate threshold function to process the wavelet coefficients $w^{j}$ and get the estimation wavelet coefficients $\widehat{w}^{j}$ :

$$
\begin{aligned}
& \widehat{w}^{j}=\operatorname{sgn}\left(w^{j}\right)\left(\left|w^{j}\right|-\lambda\right), \quad\left|w^{j}\right| \geq \lambda, \\
& \widehat{w}^{j}=0, \quad\left|w^{j}\right| \leq \lambda,
\end{aligned}
$$

where $\lambda$ is threshold, $\lambda=\sigma \sqrt{2 \log N}, \sigma$ is the mean square error of the signal, and $N$ is the sampling point number.

(3) Use the estimation wavelet coefficients $\widehat{w}^{j}$ and get the reconstruct signal $\widehat{v}$ :

$$
\widehat{v}^{j}=\widetilde{H} U \widehat{w}^{j-1}+\widetilde{G} U v^{j-1},
$$

where $\widetilde{H}$ is reconstruct low-pass filter and $\widetilde{G}$ is reconstruct high-pass filter.
Based on ICEEMD, the approximate entropy, and wavelet denoising theory, we proposed ICEEMD-De method. The method is divided into five steps to implement processing vibrational signal. The ICEEMD-De analysis process is shown in Figure 1. At first step, we sample the anchoring detection signal and take sampling signal for analysis. Then the sampling signal is decomposed with ICEEMD method. Each IMF of the signal can be obtained. At third step, we solve the approximate entropy of each IMF based on approximate entropy theory. At fourth step, the method takes the approximate entropy as the condition of whether the IMF is denoised. When approximate entropy of the IMF is higher than threshold, the wavelet denoising was processed. Finally, by means of the wavelet soft threshold denoising technique, the noise in the intrinsic mode function (IMF) is eliminated. The original signal components are retained in maximum with ICEEMD-De.

\section{Vibration Simulation Signal Analysis}

Focusing on the analysis of the anchor detection signal, one vibration simulation signal is considered, and the signal is decomposed by means of ICEEMD. The ICEEMD method is used in order to analyze the signal under the noise interference condition. The noise signal is directly decomposed by the ICEEMD, ICEEMD decomposition after the wavelet denoising, and ICEEMD-De for studying processing effect.

3.1. Vibration Signal Decompose with ICEEMD. The supposed vibration simulation signal $s$ is composed of $s_{1}$ and $s_{2}$, two sine functions (Figure 2). The expression is as follows:

$$
\begin{aligned}
& s_{1}=\sin (20 \pi t), \\
& s_{2}= \begin{cases}0.4 \times \sin (100 \pi t) & \text { when } 0.15 \leq t \leq 0.25 \\
0 & \text { other, }\end{cases} \\
& s=s_{1}+s_{2} .
\end{aligned}
$$

The simulation synthetic signal in Figure 2 is decomposed with the ICEEMD. The analysis results in Figure 3.

According to Figure 3, the ICEEMD decomposes the $s$ signal into four different IMFs, where the latter two characteristic moduli correspond to $s_{2}$ and $s_{1}$, respectively, 


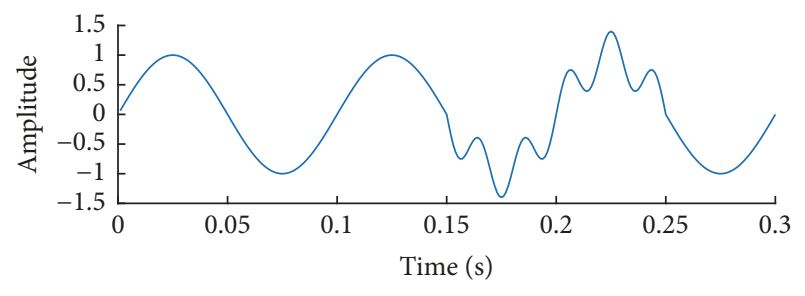

Figure 2: Vibration simulation synthetic signal.

with the corresponding frequencies of the IMF being clearly seen in regard to the spectrum. Figures $3(\mathrm{~b})$ and $3(\mathrm{~d})$ show the frequency of both $s_{2}$ and $s_{1}$, which are $100 \mathrm{~Hz}$ and $20 \mathrm{~Hz}$, respectively. Figures 3(b) and 3(d) show that the IMF1 and the IMF2 are mainly high frequency noise signals.

3.2. Vibration Signal Mode Decomposition under Noise Condition with ICEEMD. The random signal is added to the source signal in Figure 2. We take SNR $=5 \mathrm{~dB}$ as example for the analysis. The signal at $5 \mathrm{~dB}$ is analyzed by means of ICEEMD and ICEEMD-De. The results are shown in Figures 4, 5, and 6.

According to Figure 5, the IMF1 and IMF2, which were decomposed by ICEEMD, are still dominated by the random noise signal, while the IMF3 and 4 corresponding $s 1$ and $s 2$ are doped with a large number of random interference components.

From Figure 6, it can be seen that a large number of random noise interference in IMF1,2 were removed, and the IMF3, 4's corresponding $s 1, s 2$ had components of the interference significantly suppressed. The denoise signal can be clearly distinguished between the $s 1, s 2$ frequency of $100 \mathrm{~Hz}$ and $20 \mathrm{~Hz}$.

In order to further study the effect of the proposed method on the denoising of the vibration signal, the denoising effect of the wavelet and the ICEEMD-De method on the $s$ signal is compared. Figure 7 shows the error of the vibration signal after the treatment.

In Figure 7, Error 1 is the noise error, Error 2 is the error after the ICEEMD denoising, and Error 3 is the error after the wavelet denoising. From Figure 7, it can be seen that both methods are able to significantly reduce the noise interference. Based on the general trend of the error line, the proposed method results in the error line's frequency being higher, but with the error size being lower than the wavelet denoising.

In the signal denoising analysis, the signal-to-noise ratio (SNR) and the root-mean-square deviation (RMSE) are used in order to measure the denoising effect of the signal, which is defined as follows:

$$
\begin{aligned}
\mathrm{SNR} & =10 \lg \left\{\frac{\sum_{i=1}^{N} X_{i}^{2}}{\sum_{i=1}^{N}\left(X-\widehat{X}_{i}\right)^{2}}\right\}, \\
\mathrm{RMSE} & =\sqrt{\frac{\sum_{n=1}^{N}\left(X_{i}-\widehat{X}_{i}\right)^{2}}{N}} .
\end{aligned}
$$

TABLE 1: ICEEMD denoising performance index.

\begin{tabular}{lccc}
\hline Index & Original signal & ICEEMD & Wavelet \\
\hline SNR $(\mathrm{dB})$ & 5.000 & 13.741 & 11.179 \\
RMSE & 0.411 & 0.151 & 0.201 \\
\hline
\end{tabular}

According to formulas (15), two different methods can be calculated in order to denoise the effect of the index. The index is shown in Table 1.

From Table 1, we can see that both methods are able to greatly improve the SNR of the original signal, which is consistent with the results that are shown in Figure 7. The proposed method improves the SNR of the original signal by 2.7 times, while the Wavelet method increases the SNR of the original signal 2.2 times. The proposed method's RMSE is smaller than that of the wavelet method. Therefore, the ICEEMD-De denoising effect is superior to that of the wavelet denoising method.

\section{Analysis of Bolt Detection Signals}

Taking the high-slope anchor grouting test of Yunnanhighway as an example, the instrument is the LX-10 bolt, the sampling frequency is $10498 \mathrm{~Hz}$, the sampling point is 980 , and the sampling interval is $4.0 \mu$ s (Figure 8). The collected vibration signal is shown in Figure 9.

The anchor detection signals in Figure 9 are decomposed by the ICEEMD method and ICEEMD-De, respectively. The results are shown in Figures 10 and 11.

According to Figure 10, the IMF1 that is present in the high frequency noise signal, and the frequency of the IMF1 $\sim 6$ vibration modes gradually increase. In the modal IMF2, it is obvious that the noise signal can be seen. At $1.1 \mathrm{~ms}$, in the background of the bottom of the anchor reflection, the signal can be identified; however, the characteristics are not clear enough.

According to Figure 11, the noise signal in each of the modes is obviously suppressed, with the frequency of the IMF1 $\sim 6$, the vibration modes gradually decrease, and the reflection signal of the bottom of the bolt becomes clear at $1.1 \mathrm{~ms}$ in the modal IMF2. Figure 12 shows the initial reflection at $1.1 \mathrm{~ms}$ in Zoom mode, and noise interference signal was significantly suppressed. As such, the denoising effect is obvious.

\section{Conclusions}

Based on the principle of the ICEEMD decomposition, the general approximation entropy, and wavelet denoising, the ICEEMD method is introduced into the bolt detection signal analysis. The anchor detection signal is decomposed by means of using the ICEEMD, while the approximate entropy is regarded as the condition for whether or not the IMF is denoising. Using the wavelet soft threshold denoising technique to eliminate the noise in the IMF, the ICEEMDDe anchor signal analysis method is proposed. Based on the usage of the ICEEMD-De to analyze the vibration's analog signal and the anchor detection signal, the following conclusions have been drawn. 


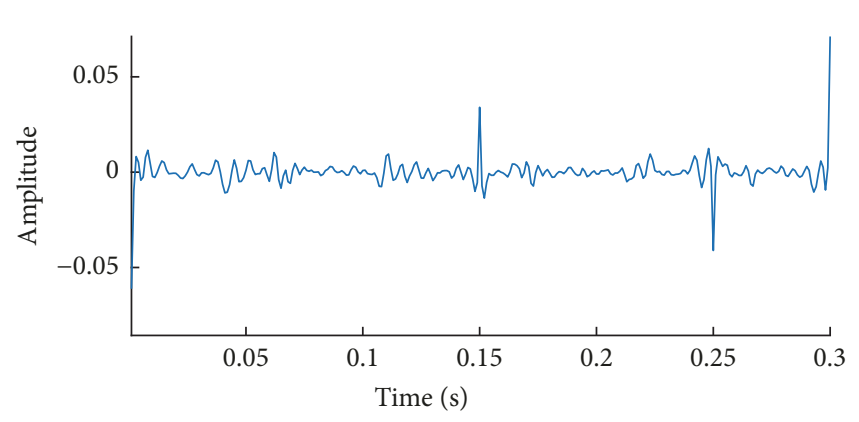

(a)

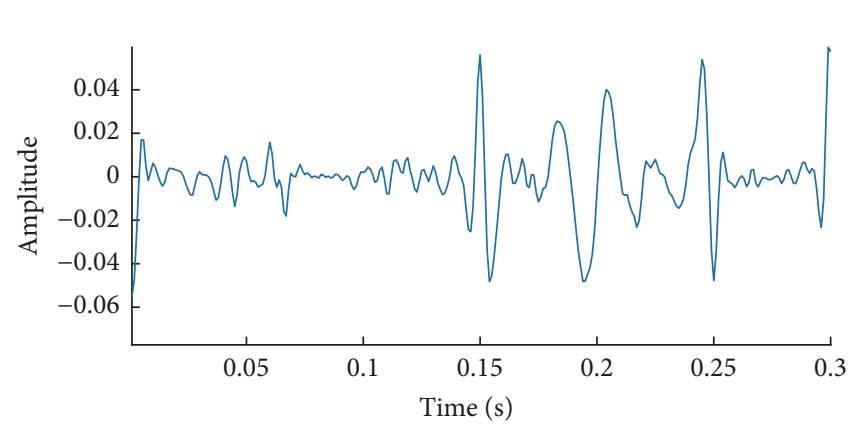

(c)

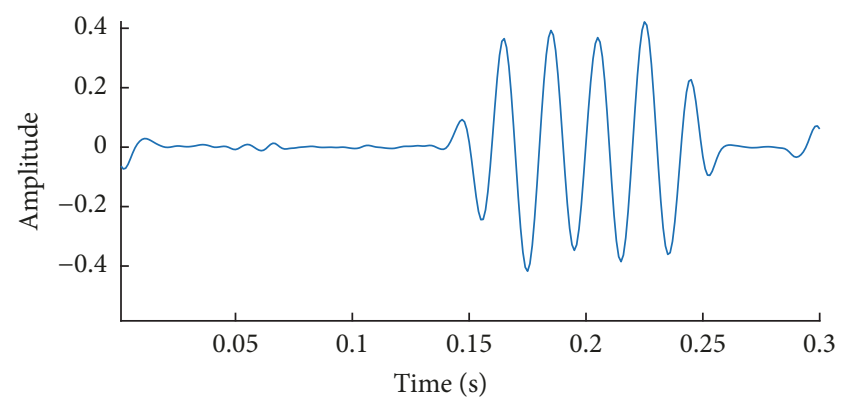

(e)

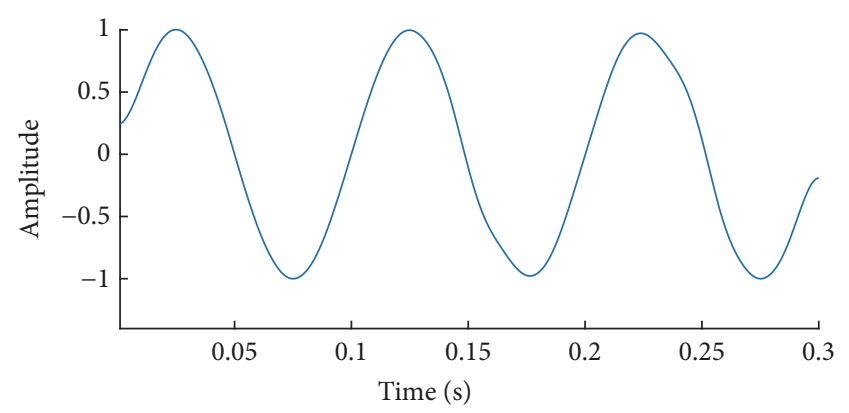

(g)

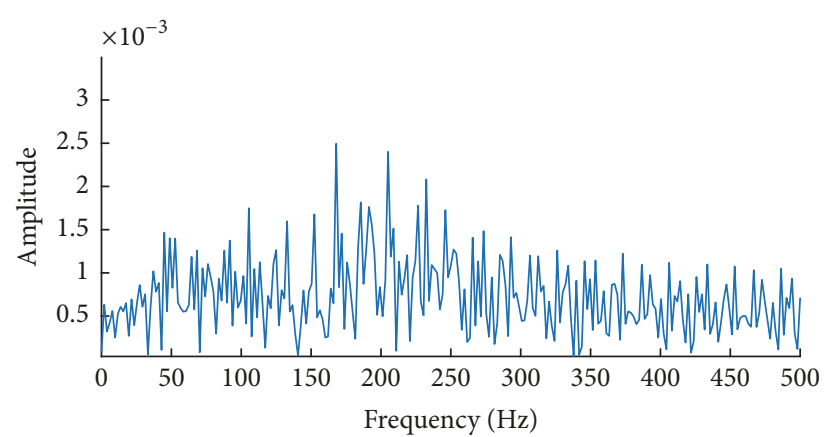

(b)

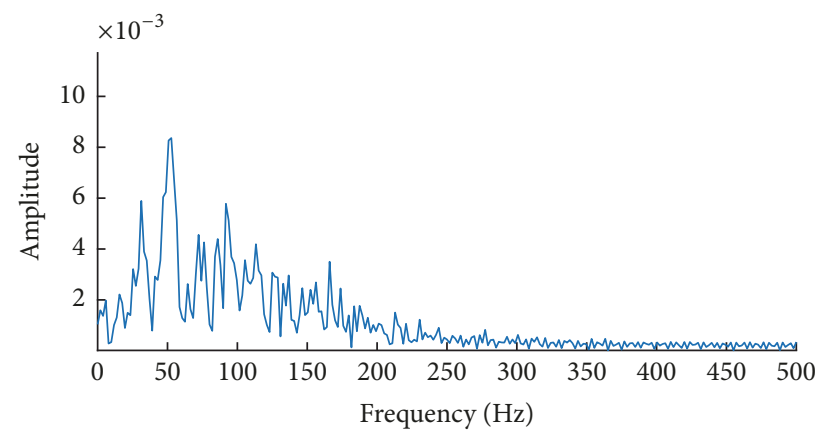

(d)

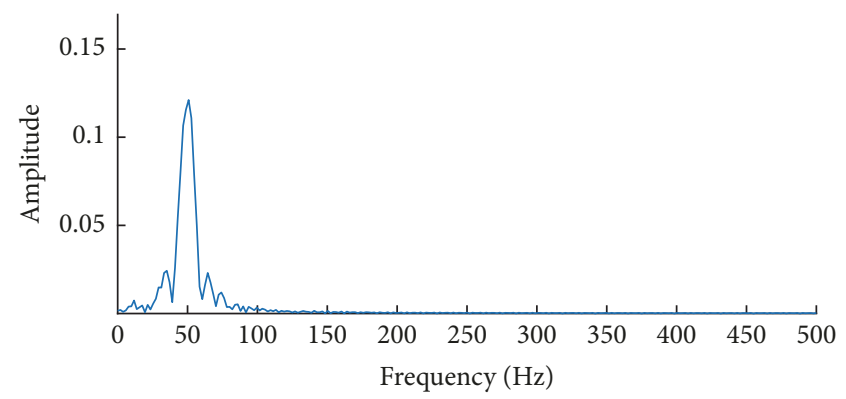

(f)

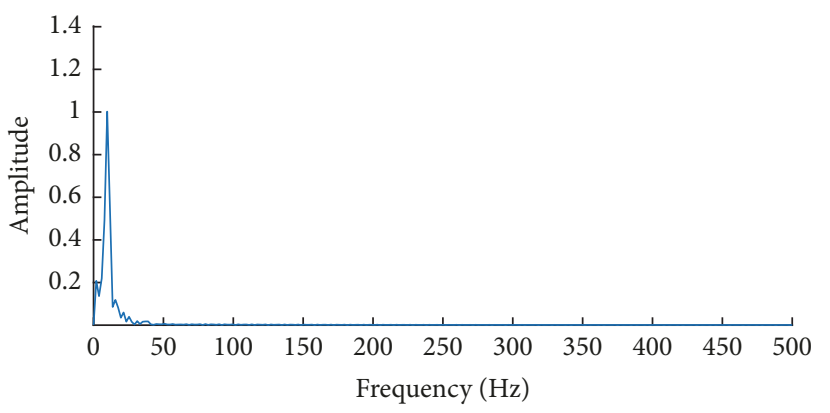

(h)

FIGURE 3: IMF and spectrum after the decomposition of the vibration signal with ICEEMD: (a, c, e, and g) IMF1 IMF4; (b, d, f, and h) IMF4 spectrum.

(1) The ICEEMD method can effectively separate the vibration modal signals to IMF.

(2) The ICEEMD-De method can effectively remove the interference in the vibration detection signal, and the denoising effect is superior to that of the traditional wavelet method.
(3) The ICEEMD method is able to separate each IMF from the bolt detection signal, and the IMF can effectively identify the bolt's end reflection time.

(4) In the analysis of the bolt detection signal, the ICEEMD-De is more effective at suppressing of the interference than the ICEEMD is. However, ICEEMD-De combine 


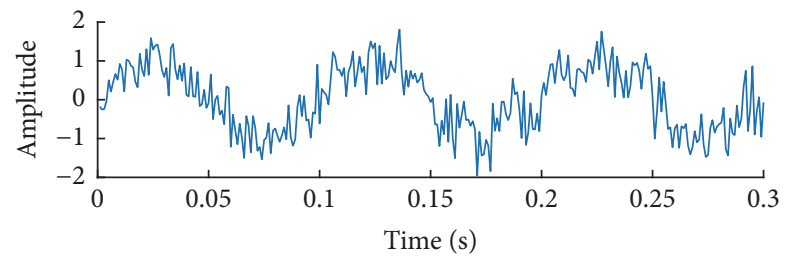

FIGURE 4: Vibration signal under noise condition.

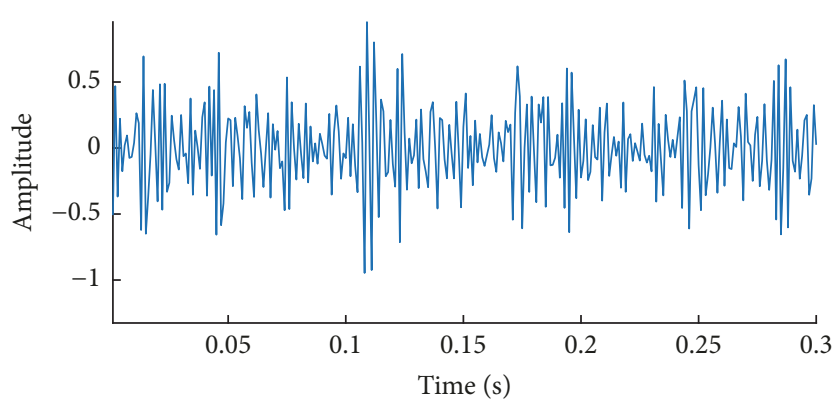

(a)

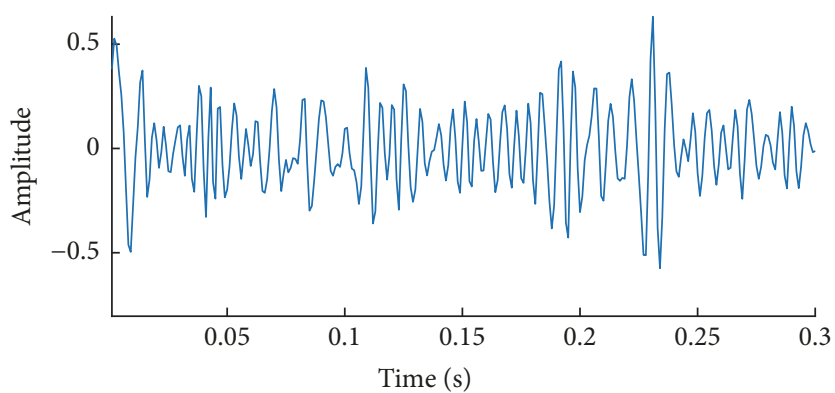

(c)

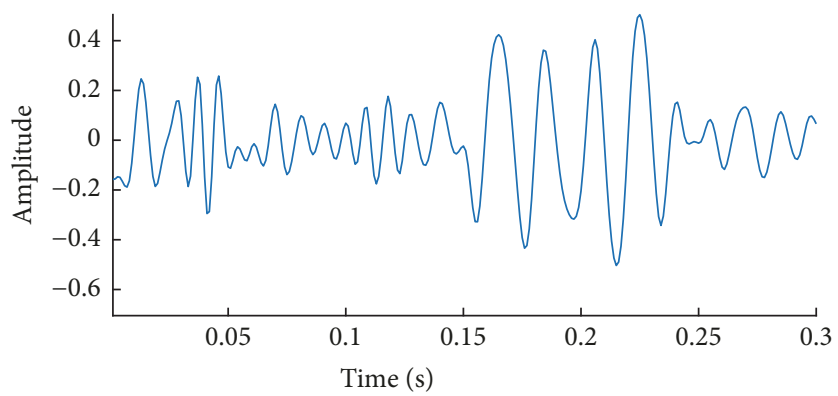

(e)

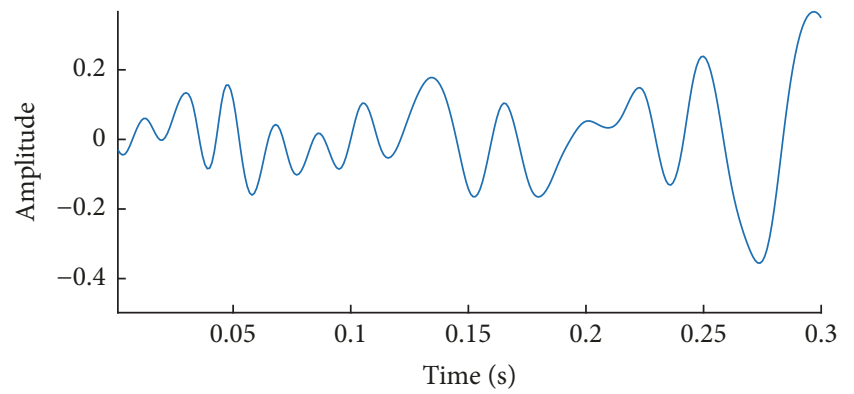

(g)

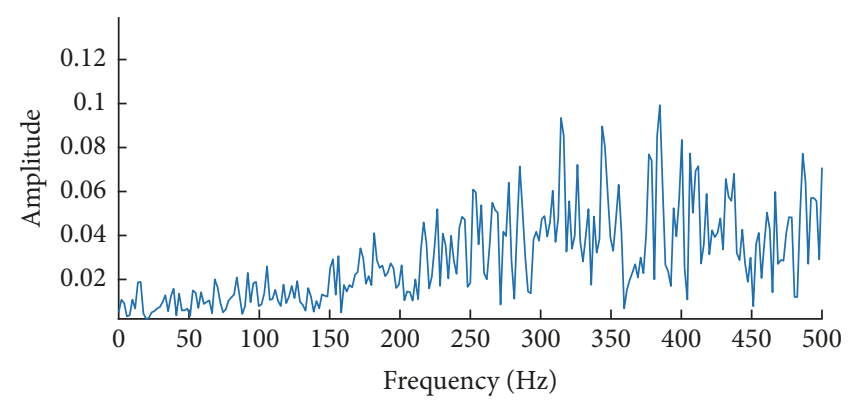

(b)

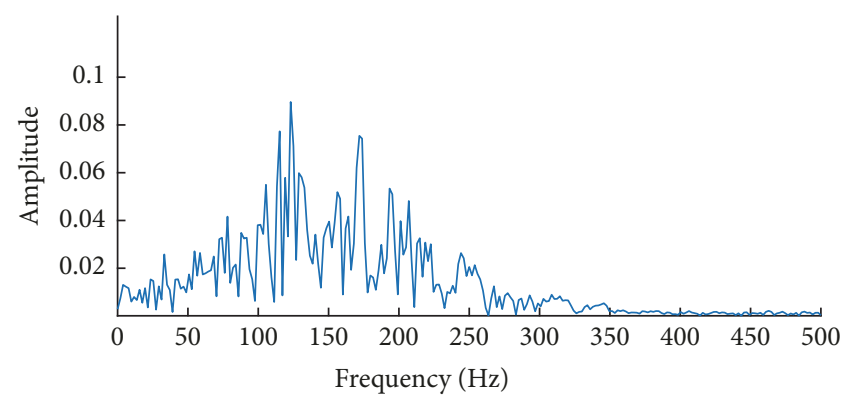

(d)

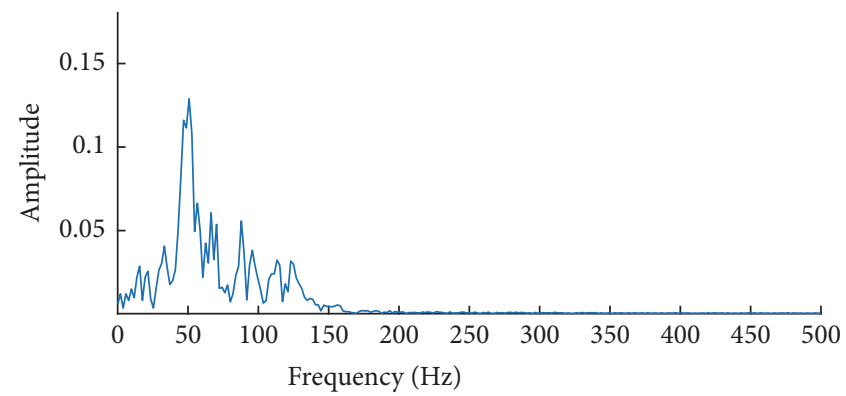

(f)

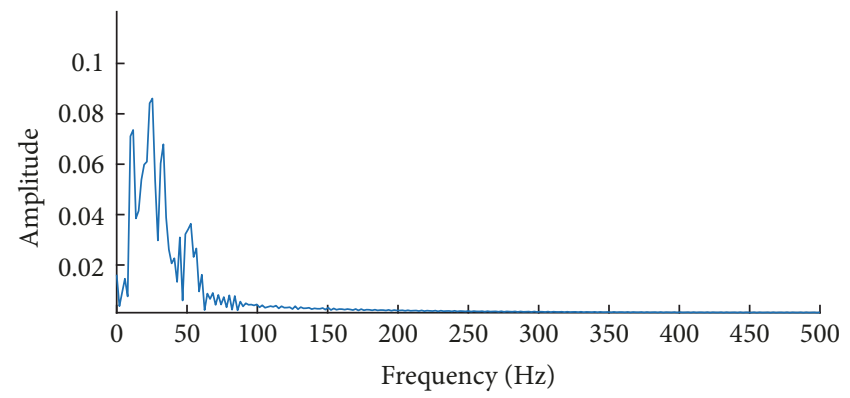

(h)

FIGURE 5: The IMF and spectrum with the vibration signal after the decomposition with ICEEMD: (a, c, e, and g) is IMF1 IMF4 and (b, d, f, and $\mathrm{h}$ ) is IMF1 IMF4 spectrum. 


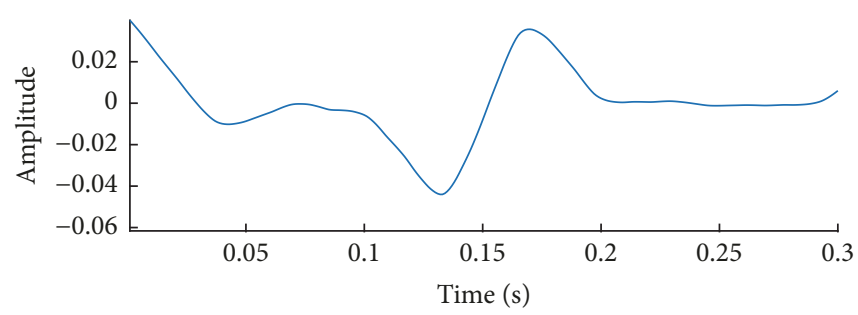

(a)

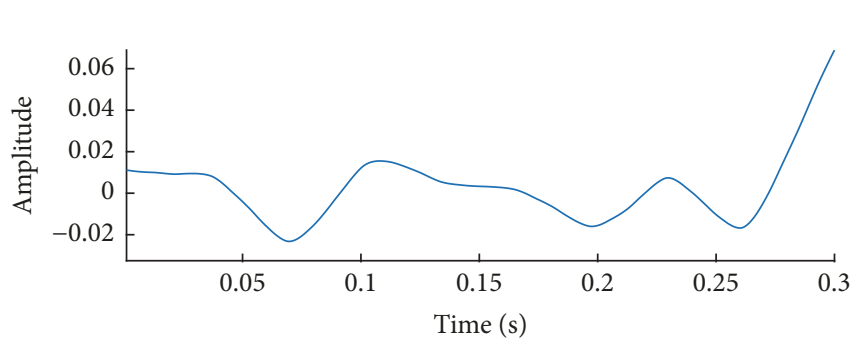

(c)

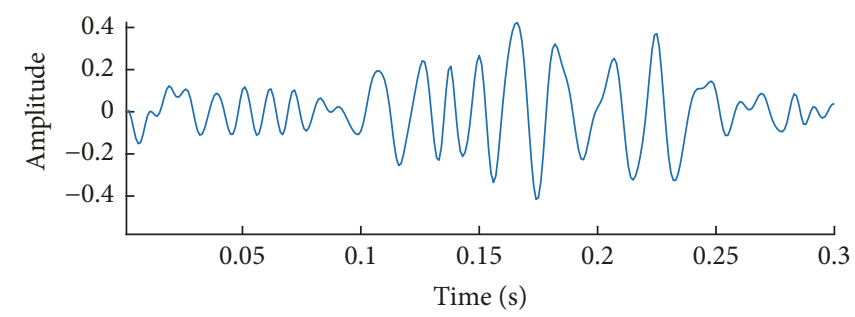

(e)

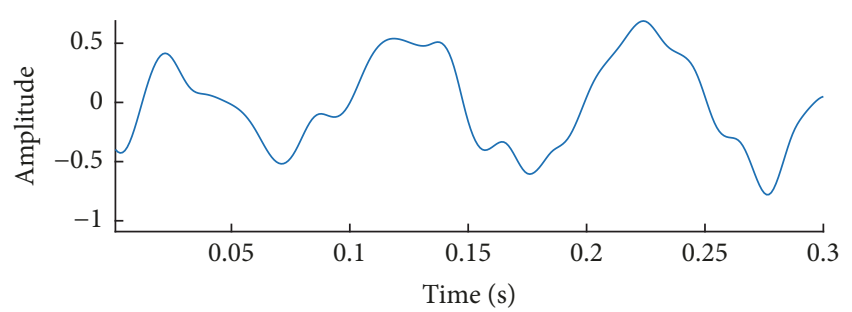

(g)

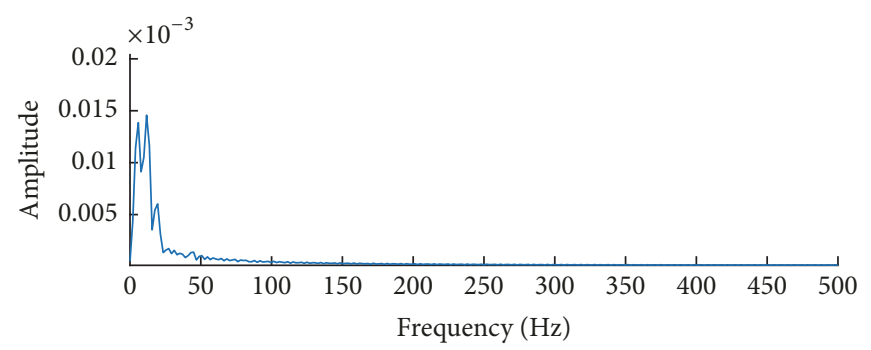

(b)

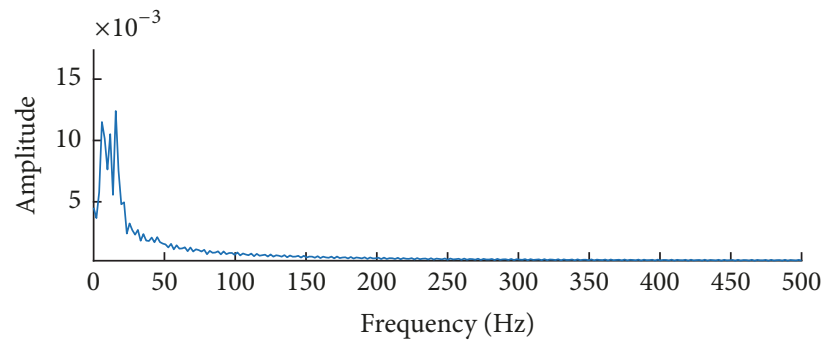

(d)

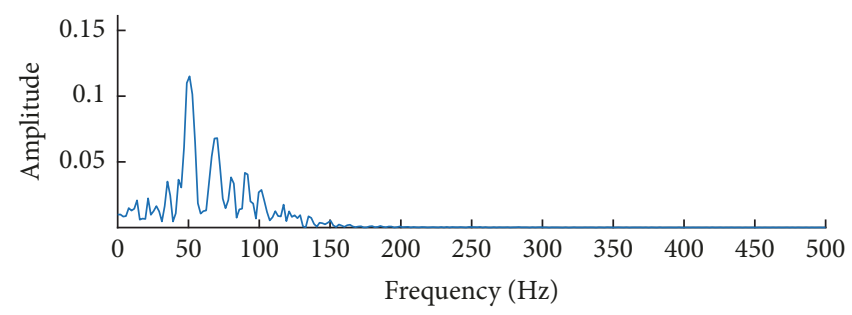

(f)

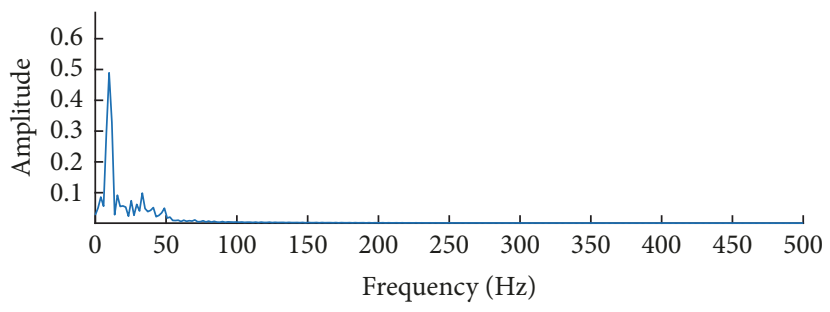

(h)

FIGURE 6: The IMF and spectrum with the vibration signal after the decomposition with ICEEMD-De: (a, c, e, and g) is IMF1 IMF4 and (b, $\mathrm{d}, \mathrm{f}$, and $\mathrm{h}$ ) is IMF1 IMF4 spectrum.

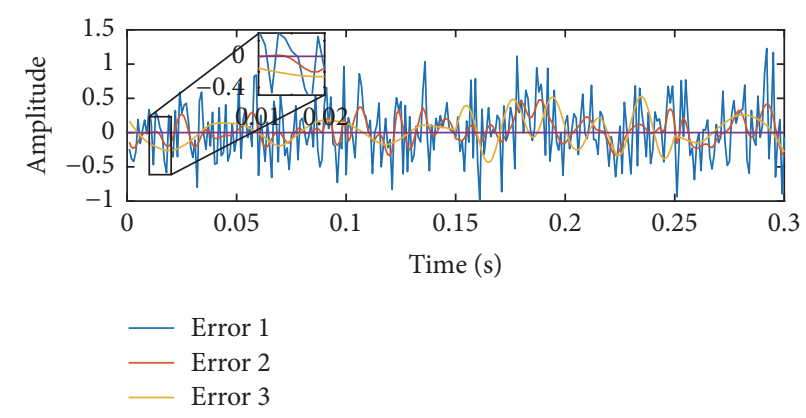

FIgURE 7: Denoising error line with wavelet and ICEEMD-De. 


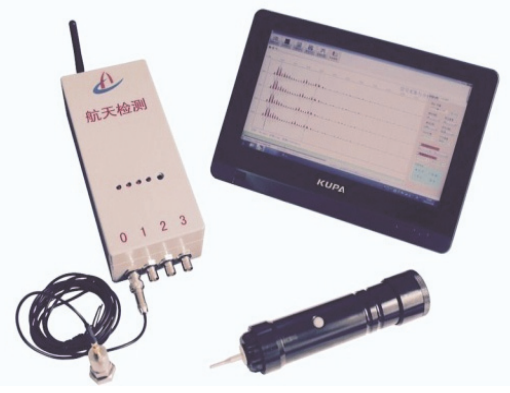

(a) Detection instrument

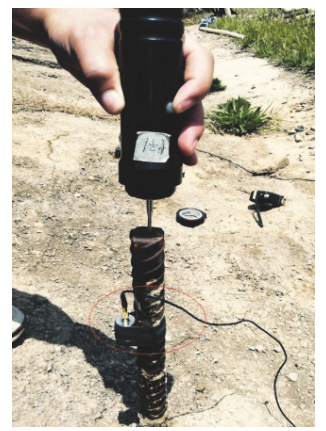

(b) Testing site

FIGURE 8: Bolt detection testing instrument and site.

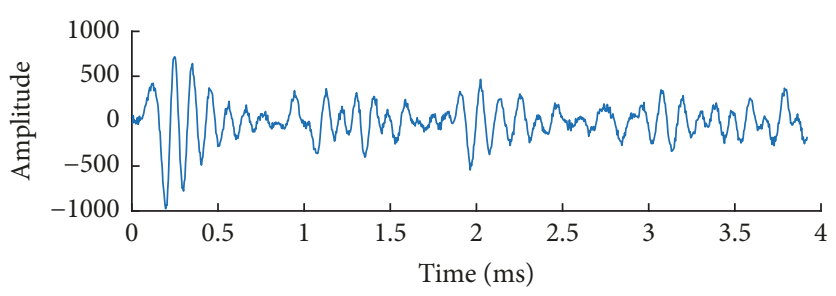

Figure 9: Bolt detection signal in actual engineering.

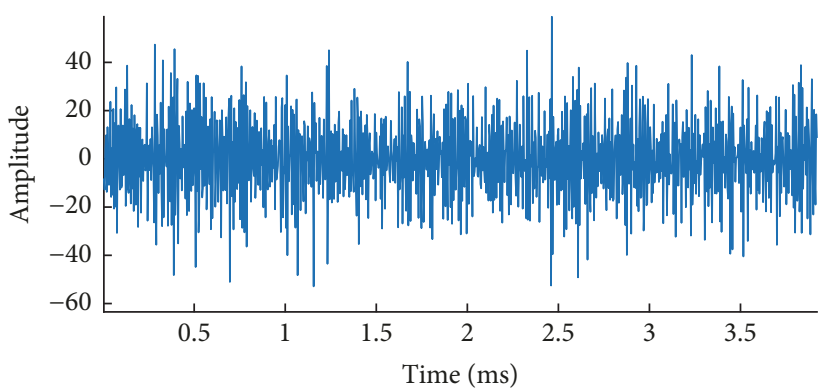

(a)

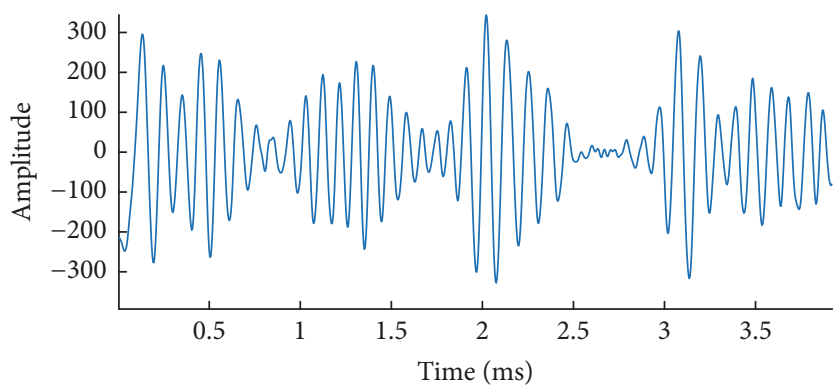

(c)

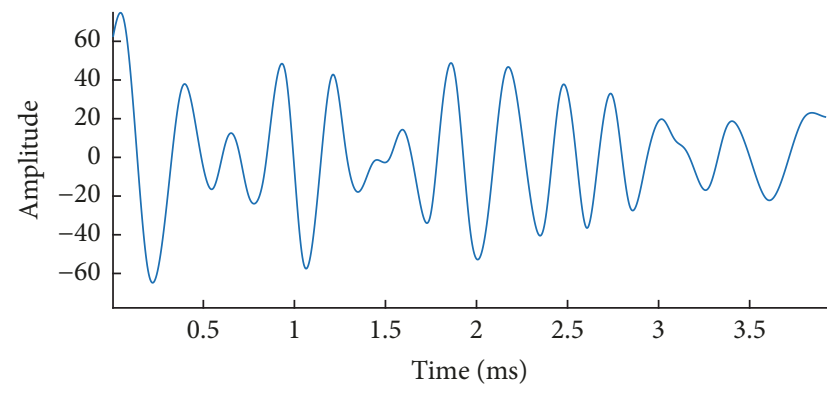

(e)

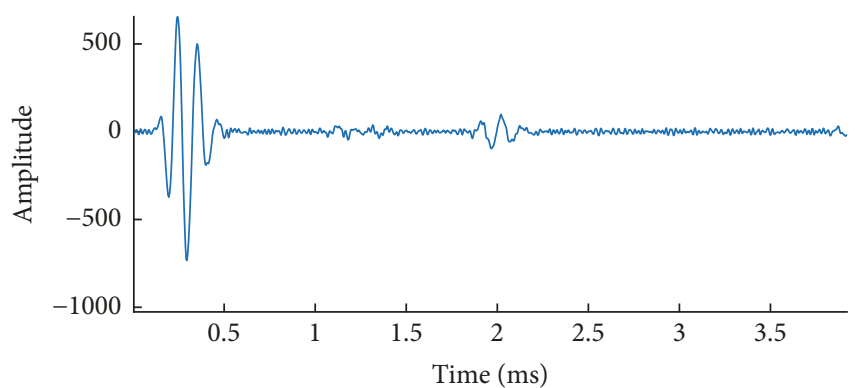

(b)

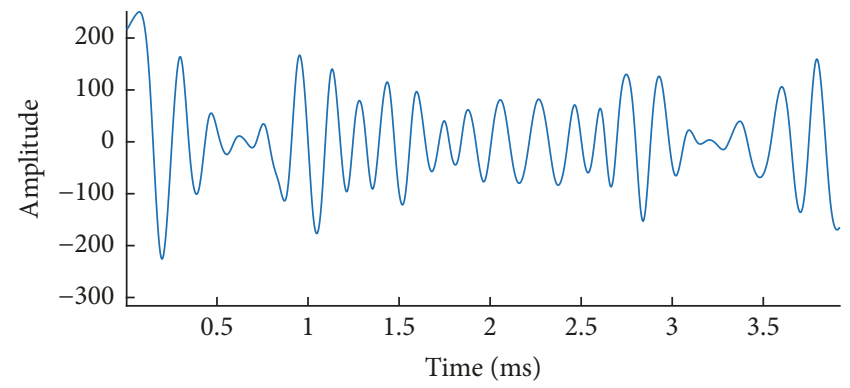

(d)

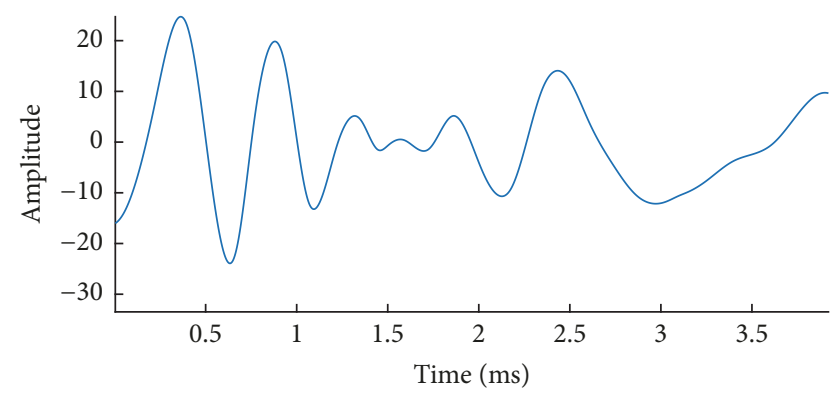

(f)

FIGURE 10: The IMF with the vibration signal after the decomposition with ICEEMD: (a) (f) show IMF1 6 Signal decomposed with ICEEMD. 


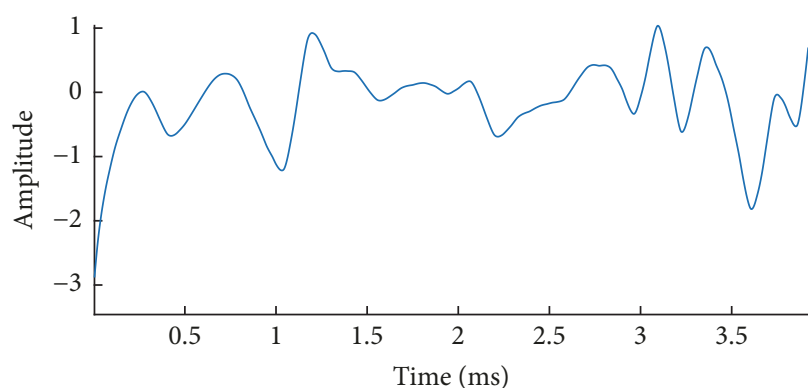

(a)

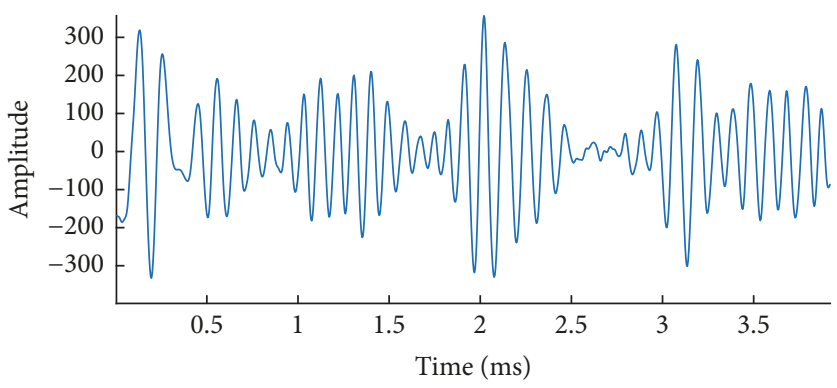

(c)

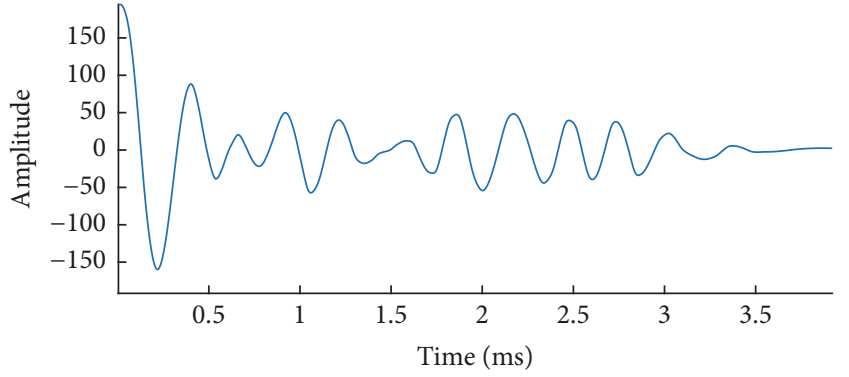

(e)

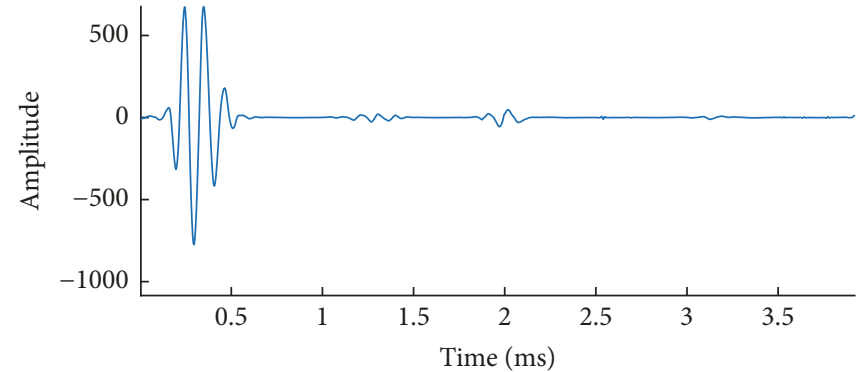

(b)

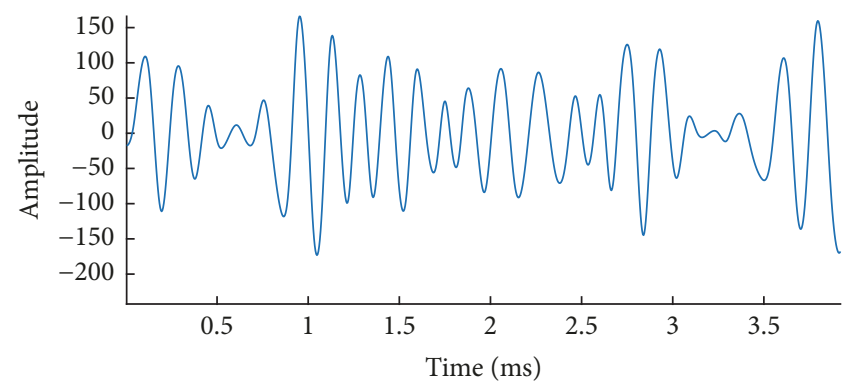

(d)

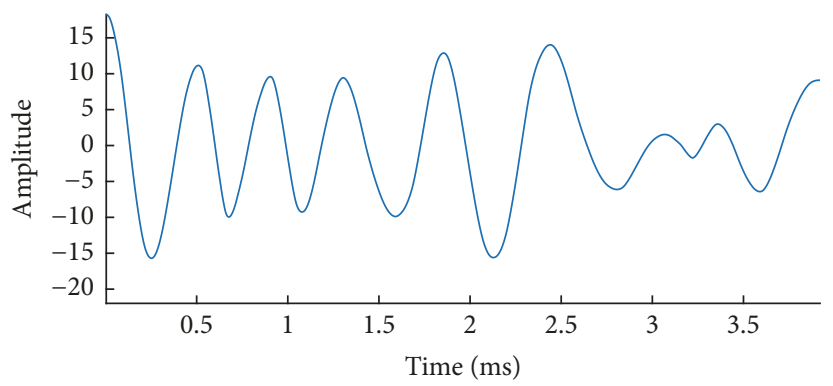

(f)

FIgURE 11: The IMF with the vibration signal after the decomposition with ICEEMD-De: (a) (f) show t IMF1 6 which is the signal decomposed with ICEEMD-De.

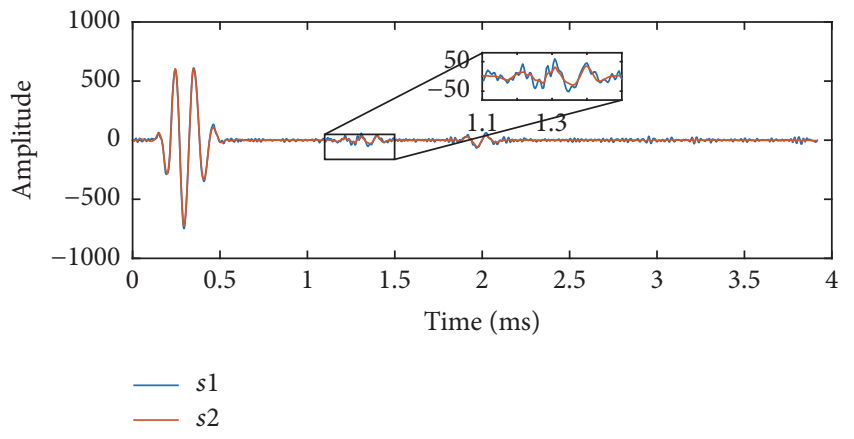

Figure 12: ICEEMD IMF2 versus ICEEMD-De IMF2.

ICEEMD and wavelet denoise technology. The method has many analysis steps. So the computational cost of the method is higher than traditional method.

\section{Conflicts of Interest}

The authors declare that they have no conflicts of interest.

\section{Acknowledgments}

This research was funded by the Open Research Fund of Key Laboratory of Hydraulic and Waterway Engineering of the Ministry of Education (Grant no. SLK2017A02) and A Project Funded by the Priority Academic Program Development of Jiangsu Higher Education Institutions (Grant no. 3014SYS1401). The authors wish to thank Su Jiankun from Yunnan Aerospace Engineering Geophysical Limited by Share Ltd. for providing the GPR practical detection data used in this study.

\section{References}

[1] B. Stimpson, "A simple rock bolt pull-out test device for teaching purposes," International Journal of Rock Mechanics and Mining Sciences, vol. 21, no. 4, pp. 217-218, 1984.

[2] U. G. Shuan-Cheng, J. Zhang, S. Zhang et al., "Influence Analysis of Anchoring Defects on Bolt Pull-out Load," Safety in Coal Mines, 2013.

[3] A. Ivanović, A. Starkey, R. D. Neilson, and A. A. Rodger, "The influence of load on the frequency response of rock bolt 
anchorage," Advances in Engineering Software, vol. 34, no. 11-12, pp. 697-705, 2003.

[4] M. D. Beard and M. J. S. Lowe, "Non-destructive testing of rock bolts using guided ultrasonic waves," International Journal of Rock Mechanics and Mining Sciences, vol. 40, no. 4, pp. 527-536, 2003.

[5] H. X. Yue, G. M. Liu, and L. I. Qi, “Development of Bolt's Testing Technique," Soil Engineering \& Foundation, 2005.

[6] S. M. Parvasi, S. C. M. Ho, Q. Kong, R. Mousavi, and G. Song, "Real time bolt preload monitoring using piezoceramic transducers and time reversal technique - A numerical study with experimental verification," Smart Materials and Structures, vol. 25, no. 8, Article ID 085015, 2016.

[7] G. Song, W. Li, B. Wang, and S. C. M. Ho, "A review of rock bolt monitoring using smart sensors," Sensors, vol. 17, no. 4, article no. 776, 2017.

[8] Q. J. Zhang, U. W. Ji-Min, G. Peng et al., "Factors in quality of special bolts based on sonic non-destructive detection," Journal of Hohai University, vol. 37, no. 2, pp. 179-184, 2009.

[9] D. Zeng H and S. Wang S, "Analysis on lateral dynamic response of anchor system[J]," Chinese Journal of Underground Space Engineering, 2010.

[10] M. Rucka and B. Zima, "Elastic wave propagation for condition assessment of steel bar embedded in mortar," International Journal of Applied Mechanics and Engineering, vol. 20, no. 1, pp. 159-170, 2015.

[11] G. Wang and B. Li, "Research on Non-destructive Testing Techniques of Bolt," Chinese Journal of Engineering Geophysics, 2009.

[12] C. Wang, W. He, J. Ning, and C. Zhang, "Propagation properties of guided wave in the anchorage structure of rock bolts," Journal of Applied Geophysics, vol. 69, no. 3-4, pp. 131-139, 2009.

[13] J. Wang, Y. Zhao, B. Yao, and J. Xu, "Filtering detecting signal of rockbolt with harmonic wavelet," Mining Science and Technology, vol. 20, no. 3, pp. 411-414, 2010.

[14] M. I. Lee, I. S. Han, J. H. Kim et al., "Evaluation of rock bolt integrity using Fourier and wavelet transforms," Tunnelling \& Underground Space Technology Incorporating Trenchless Technology Research, vol. 28, no. 28, pp. 304-314, 2012.

[15] M. Szmajda, K. Górecki, and J. Mroczka, "Gabor transform, spwvd, gabor-wigner transform and wavelet transform - tools for power quality monitoring," Metrology and Measurement Systems, vol. 17, no. 3, p. 6, 2010.

[16] J. Xu, Q. Ren, and Z. Shen, "Low strain pile testing based on synchrosqueezing wavelet transformation analysis," Journal of Vibroengineering, vol. 18, no. 2, pp. 813-825, 2016.

[17] J. Xu, Q. Ren, and Z. Shen, “1893. Ground-penetrating radar time-frequency analysis method based on synchrosqueezing wavelet transformation," Journal of Vibroengineering, vol. 18, no. 1, pp. 315-323, 2016.

[18] N. E. Huang and Z. Wu, "A review on Hilbert-Huang transform: method and its applications to geophysical studies," Reviews of Geophysics, vol. 46, no. 2, Article ID RG2006, 2008.

[19] P. Flandrin, G. Rilling, and P. Gonçalvés, "Empirical mode decomposition as a filter bank," IEEE Signal Processing Letters, vol. 11, no. 2, pp. 112-114, 2004.

[20] C. M. Wu and N. E. Huang, Biomedical Data Processing Using HHT: A Review, Springer, Berlin Heidelberg, Germany, 2009.

[21] A.-O. Boudraa and J.-C. Cexus, "EMD-based signal filtering," IEEE Transactions on Instrumentation \& Measurement, vol. 56, no. 6, pp. 2196-2202, 2007.
[22] D. Yu, J. Cheng, and Y. Yang, "Application of EMD method and Hilbert spectrum to the fault diagnosis of roller bearings," Mechanical Systems and Signal Processing, vol. 19, no. 2, pp. 259270, 2005.

[23] Y. Kopsinis and S. McLaughlin, "Development of EMD-based denoising methods inspired by wavelet thresholding," IEEE Transactions on Signal Processing, vol. 57, no. 4, pp. 1351-1362, 2009.

[24] S. Z. Lin and S. G. Wang, "EMD analysis of northern hemisphere temperature variability during last 4 centuries," Journal of Tropical Meteorology, vol. 20, no. 1, pp. 90-96, 2004.

[25] T. Wang, M. Zhang, Q. Yu, and H. Zhang, "Comparing the applications of EMD and EEMD on time-frequency analysis of seismic signal," Journal of Applied Geophysics, vol. 83, pp. 29-34, 2012.

[26] C.-S. Chen and Y. Jeng, "Natural logarithm transformed EEMD instantaneous attributes of reflection data," Journal of Applied Geophysics, vol. 95, pp. 53-65, 2013.

[27] Y. G. Lei, Z. J. He, and Y. Y. Zi, "EEMD method and WNN for fault diagnosis of locomotive roller bearings," Expert Systems with Applications, vol. 38, no. 6, pp. 7334-7341, 2011.

[28] Z. H. Wu and N. E. Huang, "Ensemble empirical mode decomposition: a noise-assisted data analysis method," Advances in Adaptive Data Analysis (AADA), vol. 1, no. 1, pp. 1-41, 2009.

[29] J. Helske and P. Luukko, "Ensemble Empirical Mode Decomposition (EEMD) and Its CompleteVariant (CEEMDAN)," International Journal of Public Health, vol. 60, no. 5, pp. 1-9, 2016.

[30] Y. Zhang and Z. Xie, "Ensemble empirical mode decomposition of impact-echo data for testing concrete structures," NDT \& E International, vol. 51, pp. 74-84, 2012.

[31] J. Li, C. Liu, Z. Zeng, and L. Chen, "GPR signal denoising and target extraction with the CEEMD method," IEEE Geoscience and Remote Sensing Letters, vol. 12, no. 8, pp. 1615-1619, 2015.

[32] M. A. Colominas, G. Schlotthauer, and M. E. Torres, "Improved complete ensemble EMD: a suitable tool for biomedical signal processing," Biomedical Signal Processing and Control, vol. 14, no. 1, pp. 19-29, 2014.

[33] W. Chen, "Ground roll attenuation using improved complete ensemble empirical mode decompoistion[J]," Journal of Seismic Exploration, p. 25, 2016.

[34] M. E. Torres, M. A. Colominas, G. Schlotthauer, and P. Flandrin, "A complete ensemble empirical mode decomposition with adaptive noise," in Proceedings of the 36th IEEE International Conference on Acoustics, Speech, and Signal Processing, pp. 41444147, Prague, Czech Republic, May 2011.

[35] L. Gan, L. Zhou, and S. M. Liu, "A de-noising method for GPR signal based on EEMD," Applied Mechanics and Materials, vol. 687-691, pp. 3909-3913, 2014.

[36] J. Xu, L. Liu, Q. Ren et al., "EEMD analysis of GPR signal in time domain," Journal of Hefei University of Technology, vol. 35, no. 5, pp. 639-642, 2015.

[37] J. Xu, Q. Ren, and L. Huang, "GPR signal analysis method based on variational mode decomposition," Journal of Hohai University, vol. 46, no. 1, pp. 78-85, 2018.

[38] X. An and J. Yang, "Denoising of hydropower unit vibration signal based on variational mode decomposition and approximate entropy," Transactions of the Institute of Measurement and Control, vol. 38, no. 3, pp. 282-292, 2016. 


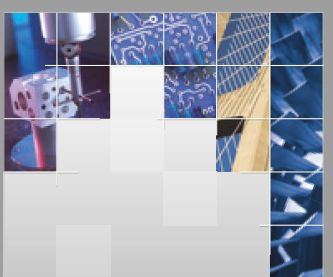

\section{Enfincering}
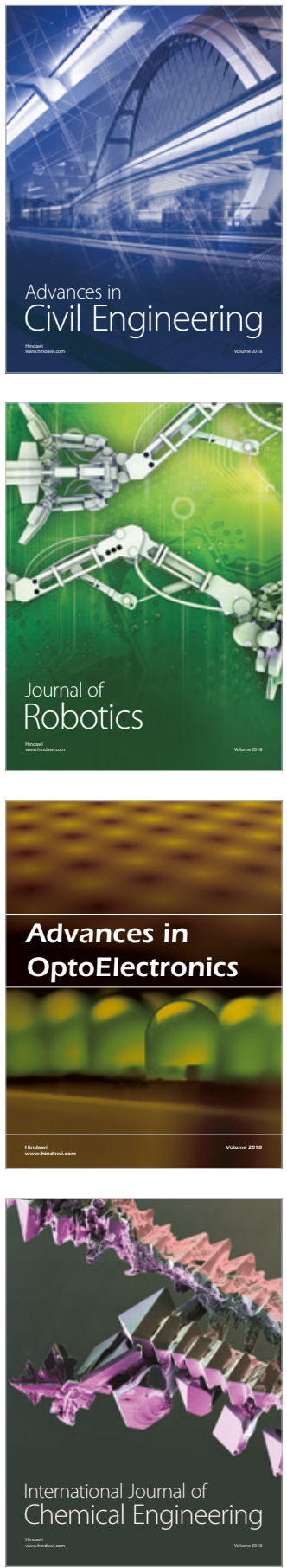

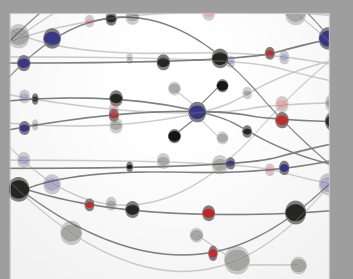

\section{Rotating \\ Machinery}

The Scientific World Journal

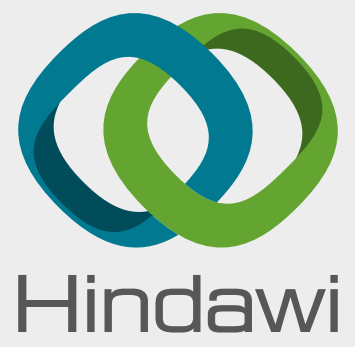

Submit your manuscripts at

www.hindawi.com
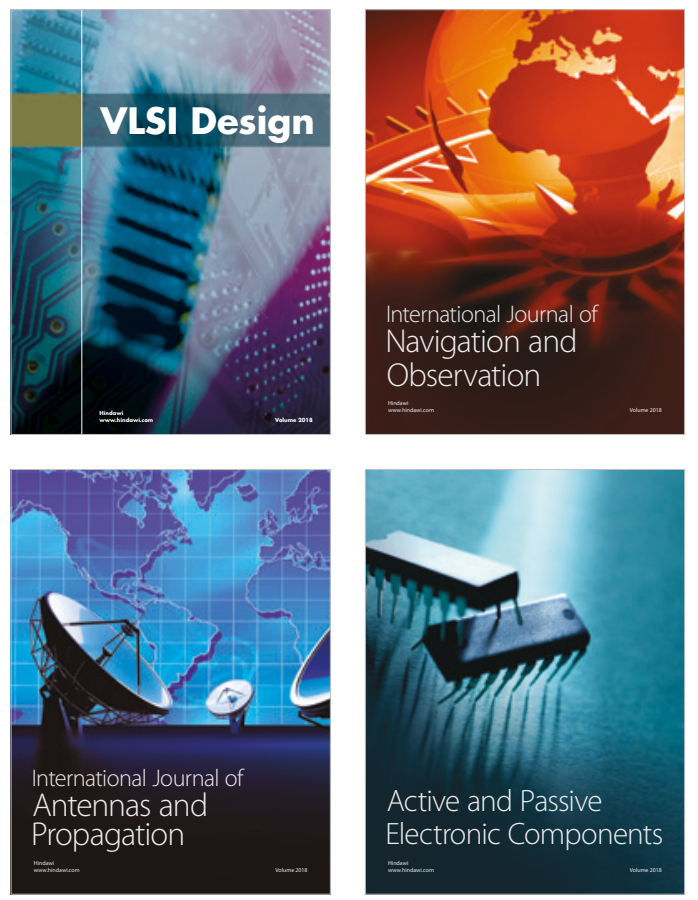
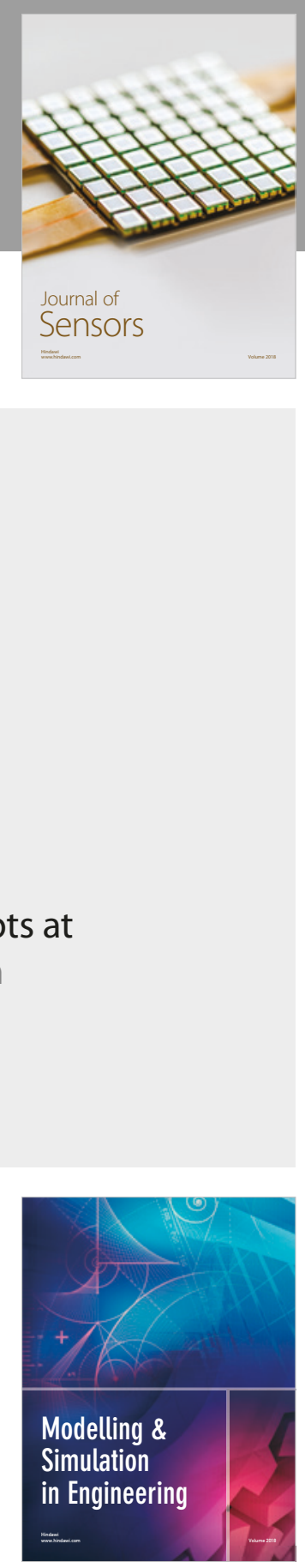

\section{Advances \\ Multimedia}
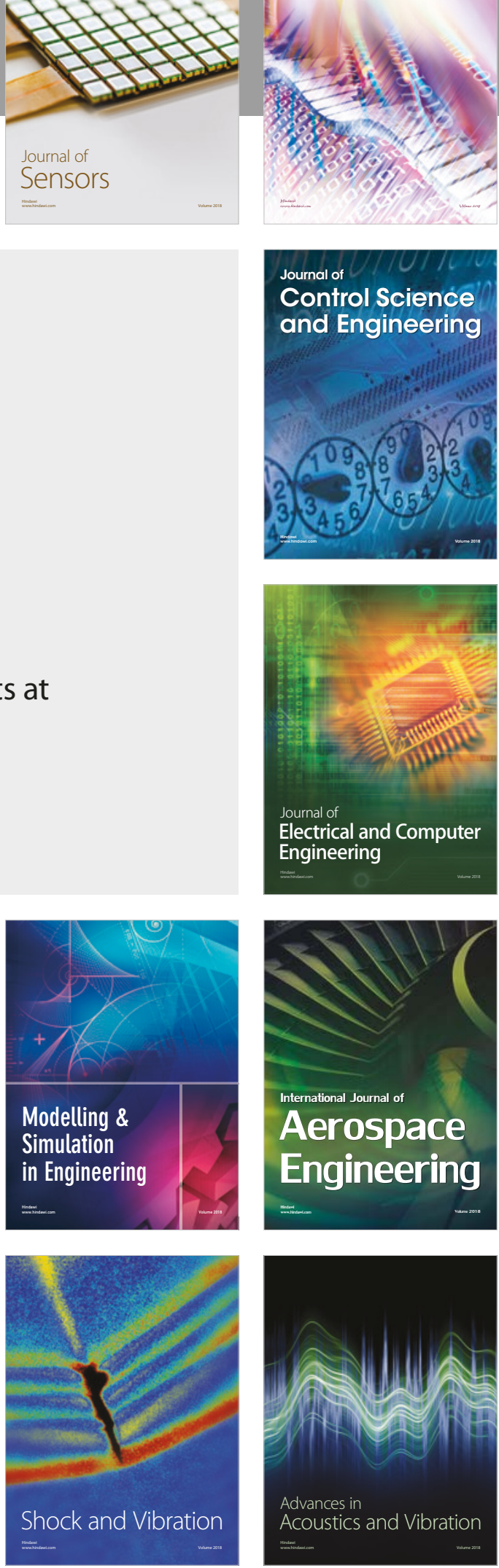